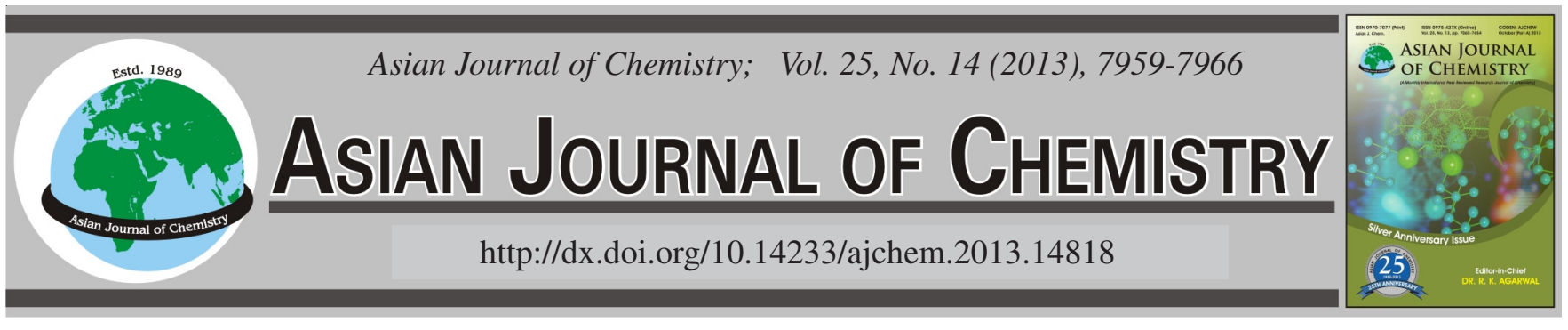

\title{
Diversified Synthesis of Novel Quinoline and Dibenzo Thiazepine Derivatives Using Known Active Intermediates
}

\section{L.N. Sharada*, G.S. Satyanarayana Reddy, B. Sammaiah and D. Sumalatha}

Department of Chemistry, Osmania University, Hyderabad-500 007, Inida

*Corresponding author: Fax: +91 40 27095171; Tel: +91 40 27152892; E-mail: dr.sharadaln@gmail.com

\begin{abstract}
The novel drug development to control resisting infections in conventional drug therapy is a need of today. Few antiulcer relative derivatives developed by approaching convergent synthesis. The derivatives synthesized successfully are dibenzo thiazepine-pyridine (SLN11-SLN15) and benzimidazole-hydroquinoline based derivatives (SLN16-SLN20). It involved the coupling through microwave, sonication and conventional techniques at final step. The efficient technology identified as sonication technique basically time and yield. The reported compounds were structural characterized by elemental analysis and spectral studies such as ${ }^{1} \mathrm{H},{ }^{13} \mathrm{C}$ NMR and MS.

Key Words: Sonication, Benzimidazole, Dibenzo thiazepine, Antiulcerative.
\end{abstract}

\section{INTRODUCTION}

For therapeutic purposes, a drug substance with wellknown chemical structure is used for developing more efficient drugs. The basic idea to prepare more analogues compounds that related drug candidates with efficient technologies. Organic molecules owe their biological activity to a variety of structural features. Sometimes a set of activities is associated with the structural backbone of a molecule.

The antiulcer drugs, omeprazole (a), is a proton pump inhibitor (PPIs) and inhibits the action of hydrogen/potassium adenosine triphosphatase $\left(\mathrm{H}^{+} / \mathrm{K}^{+}\right.$ATPase $)$in parietal cells ${ }^{1-5}$. Lansoprazole (b) is an antiulcer agent and proton pump inhibitor ${ }^{4,5}$. The structures of some antiulcer drugs available in market are given in Fig. 1.
Pantoprazole (c) suppresses the final step in gastric acid production by forming a covalent bond to two sites of the $\left(\mathrm{H}^{+}\right.$, $\mathrm{K}^{+}$)-ATPase enzyme system at the secretary surface of the gastric parietal cell ${ }^{6,7}$. Rabeprazole (d) is also demonstrated efficacy in healing and symptom relief of gastric and duodenal ulcers $^{8-10}$. Ilaprazole (e) is a proton pump inhibitor (PPI) used in the treatment of dyspepsia, peptic ulcer disease (PUD) and duodenal ulcer ${ }^{11}$. Tenatoprazole is an extended plasma halflife which makes it more effective in the treatment of nocturnal acid breakthrough than esomeprazole ${ }^{12}$.

The art has endeavoured to synthesize a variety of piperzine derivatives. Among the piperzine derivatives available as antiulcer drugs, 1-[2-(orthochloro-chlorobenzydryloxy) ethyl]-4-(ortho-methylbenzyl)piperzine well known ${ }^{13,14}$. The<smiles>COc1ccc2nc(S(=O)Cc3ncc(C)c(OC)c3C)[nH]c2c1</smiles>

Omeprazole (a)<smiles>Cc1c(OCC(F)(F)F)ccnc1CS(=O)c1nc2ccccc2[nH]1</smiles>

Lansoprazole (b)<smiles>COc1ccnc(CS(=O)c2nc3ccc(OC(F)F)cc3[nH]2)c1OC</smiles>

Pantoprazole (c)<smiles>COCCCOc1ccnc(CS(=O)c2nc3ccccc3[nH]2)c1C</smiles>

Rebepraprazole (d)

Fig. 1. Several antiulcer drugs in the market<smiles>COc1ccc2nc(S(=O)Cc3ncc(C)c(OC)c3C)[nH]c2n1</smiles>

Tenatoprazole (f) 
selection of well-known skeleton, strategic synthetic approach, technologies applied for reactions. The maximum antiulcerative drugs are prazoles. The prazoles skeleton considered for development of novel moieties into literature. The idea to incorporate the piperzine with pyridine derivatives of prazoles considered to design new skeleton (a) and benzimidazoles of different prazoles with hydroquinoline derivative.
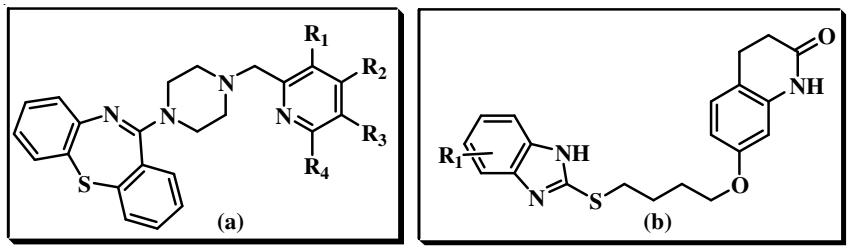

Fig. 2. Designed skeletons

A strategy of convergent synthesis, that aims to improve the efficiency of multi-step chemical synthesis, most often in organic synthesis. In linear synthesis the overall yield quickly drops with each reaction step. Here in, the synthesis of two tile derivatives and coupled considered easy and found excellent literature for easy synthesis of both ends approached convergent than linear.

The reliable technology useful for reaching target is very important to reach target very simple and cost effective. The second technology is the way of reaction conditions are using, for getting lesser reaction timings and high yield. The N-alkylation step differentiated via microwave, sonication and conventional method.

The microwave mediated organic reactions ${ }^{15}$ take place more rapidly, safely and in an environmentally friendly manner, with high yields. Very little solvent and even the use of water as a solvent is a big advantage of microwave chemistry. Recently, microwave ${ }^{16}$ and ultrasonication ${ }^{17}$ assisted synthesis in organic chemistry is quickly growing. Many organic reactions proceed much faster with higher yields under microwave irradiation compared to conventional heating. It has long been known that molecules undergo excitation with electromagnetic radiation is a technique for microwave synthesis ${ }^{18}$. Simultaneously, sonication reactions enhances the reaction rates up to a million times, believed to be due small cavities $(100 \mu)$ which implode, creating tremendous heat and pressure, shock waves and particular accelerations.

\section{EXPERIMENTAL}

All the reactions routinely monitored by thin-layer chromatography (TLC) using Merck silica gel $60 \mathrm{~F}_{254}$ coated aluminium plates using several solvent systems of different polarity. The following mobile phases were employed ethyl acetate/hexane, ethylacetate/dichloromethane, methanol/ dichloromethane and methanol-ethyl acetate with different percentage combinations. The column chromatography by using all vensil columns are used for purification of compounds used (60-120 mesh) silica-gel. The melting points were determined in open capillaries on a Thermonick melting point apparatus and found uncorrected. ${ }^{1} \mathrm{H}$ NMR (400 MHz) and ${ }^{13} \mathrm{C}$ NMR $\left(100 \mathrm{MHz}\right.$ ) recorded on $\mathrm{CDCl}_{3}$ and DMSO- $d_{6}$ solution in a $5 \mathrm{~mm}$ tube on Varian $400 \mathrm{MHz}$ Unity Inova using TMS

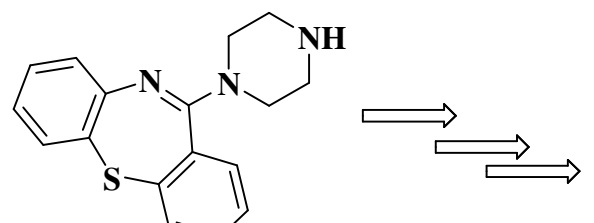

(SM1)<smiles>CN1CCN(C2=Nc3ccccc3Sc3ccccc32)CC1</smiles>

(Target)
C-N

COUPLING

via

Micro-Wave (or)

Ultra - Sonication

(or) Conventional
(Tile-1)

$+$<smiles>[R]c1ccnc(CCl)c1</smiles>

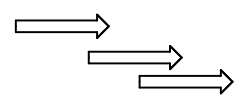

(SM2)

(Tile-2)

Scheme-I: Retro of the skeleton (a) based on convergent methodology

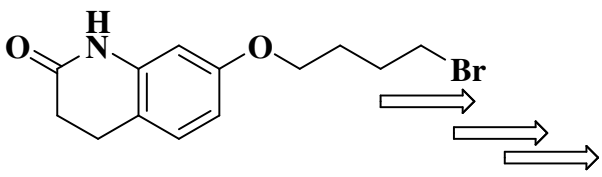

(Tile-1)<smiles>[R1]c1ccc2[nH]c(SCCCCOc3ccc4c(c3)NC(=C)CC4)nc2c1</smiles>

(Target)

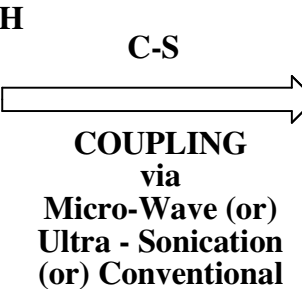<smiles>[R1]c1ccc2nc(S)[nH]c2c1</smiles>

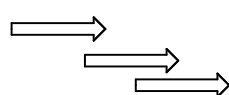

(SM2)

(Tile-2)

Scheme-II: Retro of the skeleton (b) based on convergent methodology 
internal reference standard (chemical shifts in $\delta$ ). Mass spectra were recorded on Agilent 6310 Ion Trap and Shimadzu LCMS (e/z and relative intensity). Microwave reactions are carried out in SEM Discovery (sixty). Ultra sonication reactions performed in Sonirex sonicator.

General procedure for preparation of diazathiazapinepyridine derivatives (SLN11-SLN15) and benzimidazolehydroquinoline derivatives (SLN16 - SLN20)

Conventional method (A): To a mixture of (Int-1 or Int2), (Int-3 to Int-12) and potassium carbonate in anhydrous $\mathrm{DMF}$ at room temperature. The reaction mixture was stirred at $40{ }^{\circ} \mathrm{C}$ for $5-6 \mathrm{~h}$. The reaction mixture was diluted with water and extracted product into ethyl acetate. The resultant crude product purified through silica-gel (60-120 mesh) column chromatography to afford yield (calcd. 35-52 \%) (SLN11SLN20).

Micro-wave method (B): To a mixture of (Int-1 or Int2), (Int-3 to Int-12) and potassium carbonate in anhydrous DMF at room temperature in a micro tube. The reaction mixture was stirred at $80{ }^{\circ} \mathrm{C}$ for $0.5 \mathrm{~h}, 100-200$ watts. The reaction mixture was diluted with water and extracted product into ethyl acetate. The resultant crude product purified through silica-gel (60-120 mesh) column chromatography to afford yield (calcd. 35-50 \%) (SLN11-SLN20).

Sonication method $(\mathbf{C})$ : To a mixture of (Int-1 or Int-2), (Int-3 to Int-12) and potassium carbonate in anhydrous DMF at room temperature. The reaction mixture was sonicated at $40{ }^{\circ} \mathrm{C}$ for $0.5 \mathrm{~h}$. The reaction mixture was diluted with water and extracted product into ethyl acetate. The resultant crude product purified through silica-gel (60-120 mesh) column chromatography to afford yield (calcd. 45-70 \%) (SLN11SLN20).

SLN11: ${ }^{1} \mathrm{H}$ NMR $\left(400 \mathrm{MHz}, \mathrm{CDCl}_{3}\right): \delta 2.21(\mathrm{~s}, 3 \mathrm{H})$, 2.31(s, 3H), 2.49-2.55 (m, 4H), 3.44-3.65 (m, 4H), $3.65(\mathrm{~s}$, $2 \mathrm{H}), 3.72(\mathrm{~s}, 3 \mathrm{H}), 6.69(\mathrm{t}, 1 \mathrm{H}), 7.01-7.18(\mathrm{~m}, 3 \mathrm{H}), 7.34-7.38$ $(\mathrm{m}, 2 \mathrm{H}), 7.39(\mathrm{~d}, J=1.6 \mathrm{~Hz}, 1 \mathrm{H}), 7.49(\mathrm{~d}, J=2 \mathrm{~Hz}, 1 \mathrm{H}), 8.20$ (s, 1H). ${ }^{13} \mathrm{C} \mathrm{NMR}\left(100 \mathrm{MHz}, \mathrm{CDCl}_{3}\right): \delta(\mathrm{ppm}) 11.04,13.27$, 59.84, 62.84, 122.66, 125.14, 125.31, 126.23, 127.97, 128.21, $129.04,130.70,132.06,132.02,132.12$, 134.11, 139.81, 148.58, 148.98, 155.91, 160.84, 164.078; MS (e/z): $445\left(\mathrm{M}^{+}\right)$. Anal. calcd. (\%) for $\mathrm{C}_{26} \mathrm{H}_{28} \mathrm{~N}_{4} \mathrm{OS}$ : C, 70.24; $\mathrm{H}, 6.35 ; \mathrm{N}, 12.60$; $\mathrm{O}, 3.60 ; \mathrm{S}, 7.21$. Found (\%): C, 70.31; H, 6.42; N, 12.69; O, 3.69; S, 7.29.

SLN12: ${ }^{1} \mathrm{H}$ NMR $\left(400 \mathrm{MHz}, \mathrm{CDCl}_{3}\right): \delta 2.02-2.07(\mathrm{~m}$, $2 \mathrm{H}), 2.25(\mathrm{~s}, 3 \mathrm{H}), 2.39-2.61(\mathrm{~m}, 4 \mathrm{H}), 3.01-3.32(\mathrm{~m}, 4 \mathrm{H}), 3.33$ $(\mathrm{s}, 3 \mathrm{H}), 3.52(\mathrm{t}, 2 \mathrm{H}), 3.68(\mathrm{~s}, 2 \mathrm{H}), 4.06(\mathrm{t}, 2 \mathrm{H}), 6.60(\mathrm{~d}, J=$ $6 \mathrm{~Hz}, 2 \mathrm{H}), 7.10(\mathrm{~d}, J=6.2 \mathrm{~Hz}, 2 \mathrm{H}), 7.13(\mathrm{t}, 1 \mathrm{H}), 7.19-7.32(\mathrm{~m}$, $2 \mathrm{H}), 7.39(\mathrm{~d}, J=6.2 \mathrm{~Hz}, 1 \mathrm{H}), 7.49(\mathrm{~d}, J=6.2 \mathrm{~Hz}, 1 \mathrm{H}), 8.26(\mathrm{~d}$, $J=6 \mathrm{~Hz}, 1 \mathrm{H})) .{ }^{13} \mathrm{C} \mathrm{NMR}\left(100 \mathrm{MHz}, \mathrm{CDCl}_{3}\right): \delta(\mathrm{ppm}) 10.60$, 29.39, 53.12, 58.72, 62.84, 64.86, 68.94, 105.49, 121.92, $122.63,125.3127 .96,128.18,129.02,130.06,132.05,132.12$, 134.16, 139.84, 147.40, 149.02, 156.49, 160.80, 163.27; MS $(\mathrm{e} / \mathrm{z}): 489\left(\mathrm{M}^{+}\right)$. Anal. calcd. $(\%)$ for $\mathrm{C}_{28} \mathrm{H}_{32} \mathrm{~N}_{4} \mathrm{O}_{2} \mathrm{~S}: \mathrm{C}, 68.82$; H, 6.60; N, 11.47; O, 6.55; S, 6.56. Found (\%): C, 68.89; H, $6.69 ; \mathrm{N}, 11.54 ; \mathrm{O}, 6.64 ; \mathrm{S}, 6.64$.

SLN13: ${ }^{1} \mathrm{H}$ NMR $\left(400 \mathrm{MHz}, \mathrm{CDCl}_{3}\right): \delta 2.30(\mathrm{~s}, 3 \mathrm{H}), 2.40-$ $2.80(\mathrm{~m}, 4 \mathrm{H}), 3.20-3.49(\mathrm{~m}, 4 \mathrm{H}), 4.35(\mathrm{~m}, 2 \mathrm{H}) 6.60(\mathrm{~d}, J=$ $6 \mathrm{~Hz}, 2 \mathrm{H}), 6.84(\mathrm{t}, 1 \mathrm{H}), 7.12(\mathrm{~d}, J=6.2 \mathrm{~Hz}, 1 \mathrm{H}), 7.14(\mathrm{t}, 1 \mathrm{H})$, $7.21-7.32(\mathrm{~m}, 4 \mathrm{H}), 7.39(\mathrm{~d}, J=6.2 \mathrm{~Hz}, 1 \mathrm{H}), 7.49(\mathrm{~d}, J=6.2 \mathrm{~Hz}$ $1 \mathrm{H}), 8.31(\mathrm{~d}, J=6 \mathrm{~Hz}, 1 \mathrm{H})) .{ }^{13} \mathrm{C}$ NMR $\left(100 \mathrm{MHz}, \mathrm{CDCl}_{3}\right): \delta$ (ppm) 10.61, 29.41, 53.13, 58.74, 62.85, 64.87, 68.96, 105.51, 121.94, 122.63, 122.71, 125.3127.96, 128.18, 129.02, 130.06, $132.05,132.12,134.16,139.84,147.40,149.02,156.49$, 160.80, 163.27; MS (e/z): $499\left(\mathrm{M}^{+}\right)$. Anal. calcd. (\%) for $\mathrm{C}_{26} \mathrm{H}_{25} \mathrm{~F}_{3} \mathrm{~N}_{4} \mathrm{OS}$ : C, 62.64; H, 5.05; F, 11.43; N, 11.24; O, 3.21; S, 6.43. Found (\%): C, 62.64; H, 5.05; F, 11.43; N, 11.24; O, $3.21 ; \mathrm{S}, 6.43$.

SLN14: ${ }^{1} \mathrm{H}$ NMR $\left(400 \mathrm{MHz}, \mathrm{CDCl}_{3}\right): \delta 2.31$ (s, 3H), 2.42$2.81(\mathrm{~m}, 4 \mathrm{H}), 3.21-3.49(\mathrm{~m}, 4 \mathrm{H}), 3.65(\mathrm{~s}, 2 \mathrm{H}), 3.72(\mathrm{~s}, 3 \mathrm{H})$, $6.76(\mathrm{~d}, J=6 \mathrm{~Hz}, 2 \mathrm{H}), 7.14(\mathrm{t}, 1 \mathrm{H}), 7.21-7.32(\mathrm{~m}, 4 \mathrm{H}), 7.39$ $(\mathrm{d}, J=6.2 \mathrm{~Hz}, 1 \mathrm{H}), 7.49(\mathrm{~d}, J=6.2 \mathrm{~Hz}, 1 \mathrm{H}), 8.23(\mathrm{~d}, J=6 \mathrm{~Hz}$, 1H) ). ${ }^{13} \mathrm{C}$ NMR $\left(100 \mathrm{MHz}, \mathrm{CDCl}_{3}\right): \delta$ (ppm) 59.84, 53.13, $58.74,62.84,62.85,64.87,68.96,105.53,121.95,122.64$, 122.72 , 125.82, 127.96, 128.18, 129.12, 130.08, 132.07, $132.15,134.19,139.88,147.48,149.08,156.51,160.83$, 163.31; MS (e/z): $431\left(\mathrm{M}^{+}\right)$. Anal. calcd. (\%) for $\mathrm{C}_{25} \mathrm{H}_{26} \mathrm{~N}_{4} \mathrm{OS}$ : C, 69.74; H, 6.09; N, 13.01; O, 3.72; S, 7.45. Found (\%): C, 69.83; H, 6.17; N, 13.05; O, 3.83; S, 7.53.

SLN15: ${ }^{1} \mathrm{H}$ NMR (400 MHz, $\left.\mathrm{CDCl}_{3}\right): \delta$ 2.50-2.57 (m, $4 \mathrm{H}), 3.46-3.68(\mathrm{~m}, 4 \mathrm{H}), 3.85(\mathrm{~s}, 2 \mathrm{H}), 3.87(\mathrm{~s}, 3 \mathrm{H}), 388(\mathrm{~s}$, $3 \mathrm{H}), 6.67(\mathrm{~d}, J=5.6 \mathrm{~Hz}, 1 \mathrm{H}), 6.84-6.88(\mathrm{~m}, 2 \mathrm{H}), 7.04-7.07$ (m, 2H), 7.34-7.38 (m, 2H), 7.38 (d, J=1.6 Hz, 1H), 7.49 (d, $J=2 \mathrm{~Hz}, 1 \mathrm{H}), 8.29$ (d, $J=6 \mathrm{~Hz}, 1 \mathrm{H}) .{ }^{13} \mathrm{C} \mathrm{NMR}(100 \mathrm{MHz}$, $\mathrm{CDCl}_{3}$ ): $\delta$ (ppm) 10.55, 53.13, 55.33, 62.80, 104.74, 121.94, $122.66,125.34,127.99,128.18,129.05,130.66,132.08$, 132.14, 134.19, 139.90, 147.47, 149.02, 156.48, 160.83, 163.98; $\mathrm{MS}(\mathrm{e} / \mathrm{z})$ : $447\left(\mathrm{M}^{+}\right)$. Anal. calcd. (\%) for $\mathrm{C}_{25} \mathrm{H}_{26} \mathrm{~N}_{4} \mathrm{O}_{2} \mathrm{~S}$ : C, 67.24; H, 5.87; N, 12.55; O, 7.17; S, 7.18. Found (\%): C, 67.28; H, 5.94; N, 12.62; O, 7.25; S, 7.243.

SLN16: ${ }^{1} \mathrm{H}$ NMR (400 MHz, $\mathrm{CDCl}_{3}$ ): $\delta$ 1.62-1.84 (m, $4 \mathrm{H}), 2.53(\mathrm{t}, 2 \mathrm{H}), 2.75(\mathrm{t}, 2 \mathrm{H}), 3.22(\mathrm{t}, 2 \mathrm{H}), 3.64(\mathrm{t}, 2 \mathrm{H}), 6.25$ $(\mathrm{t}, 1 \mathrm{H}), 6.35(\mathrm{t}, 1 \mathrm{H}), 6.75(\mathrm{~m}, 2 \mathrm{H}), 6.89(\mathrm{~d}, J=8.4 \mathrm{~Hz}, 1 \mathrm{H})$, $7.00(\mathrm{~s}, 1 \mathrm{H}), 7.42(\mathrm{~d}, J=8.8 \mathrm{~Hz}, 1 \mathrm{H}), 8.29(\mathrm{~s}, 1 \mathrm{H}), 9.61(\mathrm{~s}$, 1H). ${ }^{13} \mathrm{C}$ NMR $\left(100 \mathrm{MHz}, \mathrm{CDCl}_{3}\right): \delta$ (ppm) 24.38, 26.35, 28.05, 30.94, 31.00, 32.57, 67.24, 102.19, 109.14, 111.45, 115.66, 128.59, 138.07, 149.63, 156.12, 158.37, 172.94; MS (e/z): $368\left(\mathrm{M}^{+}\right)$. Anal. calcd. (\%) for $\mathrm{C}_{20} \mathrm{H}_{21} \mathrm{~N}_{3} \mathrm{O}_{2} \mathrm{~S}: \mathrm{C}, 65.37$; $\mathrm{H}, 5.76$; N, 11.44; O, 8.71; S, 8.73. Found (\%): C, 65.42; H, $5.81 ; \mathrm{N}, 11.47 ; \mathrm{O}, 8.75 ; \mathrm{S}, 8.75$.

SLN17: ${ }^{1} \mathrm{H}$ NMR (400 MHz, $\left.\mathrm{CDCl}_{3}\right): \delta$ 1.84-1.85 (m, 4H), 2.61 (brs, 2H), 2.82 (brs, 2H), 2.83 (brs, 2H), 3.31 (brs, 2H), 6.25-6.27 (brm, 2H), 6.41 (brs, 2H), 6.95 (brs, 2H), 7.32 (brs, 2H), 9.77(brs, $1 \mathrm{H}) .{ }^{13} \mathrm{C} \mathrm{NMR}\left(100 \mathrm{MHz}, \mathrm{CDCl}_{3}\right): \delta$ (ppm) 24.31, 26.31, 28.05, 30.97, 32.28, 67.28, 102.33, 109.14, 113.97, 115.04, 115.78, 116.55, 119.12, 128.64, 137.99, 146.69, 152.33, 158.37, 173.18; MS (e/z): $434\left(\mathrm{M}^{+}\right)$. Anal. calcd. (\%) for $\mathrm{C}_{21} \mathrm{H}_{21} \mathrm{~F}_{2} \mathrm{~N}_{3} \mathrm{O}_{3} \mathrm{~S}: \mathrm{C}, 58.19 ; \mathrm{H}, 4.88 ; \mathrm{F}, 8.77 ; \mathrm{N}$, 9.69; O, 11.07; S, 7.40. Found (\%): C, 58.23; H, 4.93; F, 8.82; N, 9.73; O, 11.13; S, 7.52.

SLN18: ${ }^{1} \mathrm{H}$ NMR (400 MHz, $\left.\mathrm{CDCl}_{3}\right): \delta$ 1.84-1.89 (m, $4 \mathrm{H}), 2.62(\mathrm{t}, 2 \mathrm{H}), 2.84(\mathrm{t}, 2 \mathrm{H}), 3.32(\mathrm{t}, 2 \mathrm{H}), 3.82(\mathrm{t}, 2 \mathrm{H}), 3.91(\mathrm{~s}$, $3 \mathrm{H}), 6.27(\mathrm{~s}, 1 \mathrm{H}), 6.37(\mathrm{~s}, 1 \mathrm{H}), 6.44(\mathrm{~m}, 1 \mathrm{H}), 6.57$ (d, $J=8.4$ $\mathrm{Hz}, 1 \mathrm{H}), 6.75(\mathrm{~d}, J=8.4 \mathrm{~Hz}, 1 \mathrm{H}), 6.96(\mathrm{~d}, J=8.4 \mathrm{~Hz}, 1 \mathrm{H}), 7.72$ $(\mathrm{d}, J=8.8 \mathrm{~Hz}, 1 \mathrm{H}), 9.35(\mathrm{~s}, 1 \mathrm{H}) .{ }^{13} \mathrm{C} \mathrm{NMR}\left(100 \mathrm{MHz}, \mathrm{CDCl}_{3}\right)$ : $\delta$ (ppm) 24.43, 26.24, 28.05, 30.94, 31.00, 32.12, 53.96, 67.37, 102.40, 105.04, 109.08, 115.759, 128.577, 138.07, 158.42, 160.94, 172.76; MS (e/z): $398\left(\mathrm{M}^{+}\right)$. Anal. calcd. (\%) for 


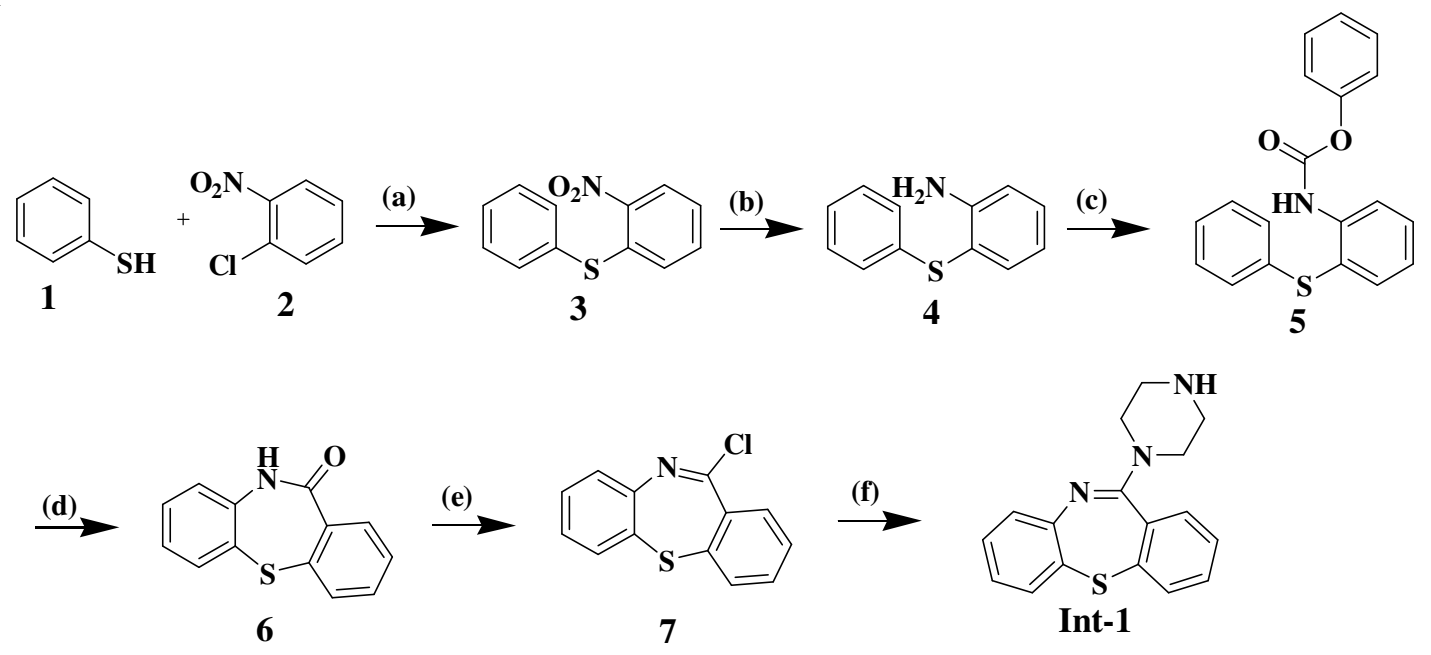

Reaction \& Conditions: (a) $\mathrm{NaOH}, \mathrm{DMSO}, 80^{\circ} \mathrm{C}$ (b) $\mathrm{Ra} \mathrm{Ni}, \mathrm{MeOH}, \mathrm{H}_{2}, 80 \mathrm{PSI}$ (c) Phenyl chloroformate, $\mathrm{Na}_{2} \mathrm{CO}_{3}, \mathrm{THF}, \mathrm{H}_{2} \mathrm{O}$ (d) $\mathrm{PPA}, 100^{\circ} \mathrm{C}$ (e) $\mathrm{POCl} 3, \mathrm{~N}, \mathrm{~N}^{\prime}$-dimethyl aniline $\mathrm{DCM}$ (f) Piperzine, Toluene/THF, triehtylamine.

Scheme-III: Synthesis of 11-pyrazolyldibenzo[b,f][1,4]thiazepine (Int-1)

$\mathrm{C}_{20} \mathrm{H}_{22} \mathrm{~N}_{4} \mathrm{O}_{3} \mathrm{~S} \mathrm{C}_{21} \mathrm{H}_{23} \mathrm{~N}_{3} \mathrm{O}_{3} \mathrm{~S}$ : C, 63.45; H, 5.83; N, 10.57; O, 12.08; S, 8.07. Found (\%): C, 63.51; H, 5.92; N, 10.63; O, $12.15 ; \mathrm{S}, 8.15$.

SLN19: ${ }^{1} \mathrm{H}$ NMR (400 MHz, $\left.\mathrm{CDCl}_{3}\right): \delta 1.60-1.85(\mathrm{~m}$, $4 \mathrm{H}), 2.57(\mathrm{t}, 2 \mathrm{H}), 2.79(\mathrm{t}, 2 \mathrm{H}), 3.24(\mathrm{t}, 2 \mathrm{H}), 3.66(\mathrm{t}, 2 \mathrm{H}), 3.71(\mathrm{~s}$, $3 \mathrm{H}), 6.29(\mathrm{~s}, 1 \mathrm{H}), 6.37(\mathrm{~d}, 1 \mathrm{H}), 6.77(\mathrm{~d}, J=8.4 \mathrm{~Hz}, 1 \mathrm{H}), 6.91$ $(\mathrm{d}, J=8.4 \mathrm{~Hz}, 1 \mathrm{H}), 7.01(\mathrm{~s}, 1 \mathrm{H}), 7.43(\mathrm{~d}, J=8.8 \mathrm{~Hz}, 1 \mathrm{H}), 9.61(\mathrm{~s}$, 1H). $\left.{ }^{13} \mathrm{C} \mathrm{NMR} \mathrm{(100} \mathrm{MHz,} \mathrm{CDCl}_{3}\right): \delta$ (ppm) 24.38, 26.35, 28.05, 30.94, 31.00, 32.57, 55.82, 67.24, 102.19, 109.14, $111.45,115.66,128.59,138.07,149.63,156.12,158.37$, 172.94; MS (e/z): $399\left(\mathrm{M}^{+}\right)$. Anal. calcd. (\%) for $\mathrm{C}_{20} \mathrm{H}_{22} \mathrm{~N}_{4} \mathrm{O}_{3} \mathrm{~S}$ : C, 60.28; H, 5.56; N, 14.06; O, 12.05; S, 8.05. Found (\%): C, $60.35 ; \mathrm{H}, 5.65 ; \mathrm{N}, 14.12 ; \mathrm{O}, 12.14 ; \mathrm{S}, 8.11$.

SLN20: ${ }^{1} \mathrm{H}$ NMR (400 MHz, $\left.\mathrm{CDCl}_{3}\right): \delta 1.84-1.88(\mathrm{~m}$, 4H), 2.60 (brs, 2H), 2.83 (brs, 2H), 2.85 (brs, 2H), 3.33 (brs, 2H), 6.02 (brs, 2H), 6.25-6.27 (brm, 2H), 6.41 (brs, 2H), 6.71 (brs, 2H) 6.95 (brs, 2H), 7.32 (brs, 1H), 9.77(brs, 1H). ${ }^{13} \mathrm{C}$ NMR (100 MHz, $\mathrm{CDCl}_{3}$ ): $\delta$ (ppm) 24.28, 26.29, 28.00, 30.92, $32.23,67.24,102.31,208.19,109.10,113.90,115.02,115.71$, 116.51, 117. 21, 119.10, 128.58, 137.96, 146.67, 152.36, 158.39, 173.19; MS (e/z): $433\left(\mathrm{M}^{+}\right)$. Anal. calcd. (\%) for $\mathrm{C}_{24} \mathrm{H}_{24} \mathrm{~N}_{4} \mathrm{O}_{2} \mathrm{~S}$ : C, 66.64; H, 5.59; N, 12.95; O, 7.40; S, 7.41. Found (\%): C, 66.64; H, 5.59; N, 12.95; O, 7.40; S, 7.41.

\section{RESULTS AND DISCUSSION}

In continuation of our research interest was to synthesize a series of novel (SLN11-SLN20) relevant to antiulcer related drug skeleton candidates prepared. The main focus in this paper described the synthesis and rest of the activity studies is under progress.

In the synthesis of Int-1, was followed the some earlier reported work $^{18}$. The coupling of $\mathbf{1}$ and $\mathbf{2}$ using $\mathrm{NaOH}$ in DMSO at $80^{\circ} \mathrm{C}$. The reduction of compound 3 with $\mathrm{Ra} \mathrm{Ni}, \mathrm{H}_{2}$ in methanol into 4 . The carbamate 5 was prepared by protection of amine 4 using phenyl chloroformate and $\mathrm{Na}_{2} \mathrm{CO}_{3}$. The cyclisation of carbamate $\mathbf{5}$ into compound $\mathbf{6}$ was carried out in presence of PPA at $80-90{ }^{\circ} \mathrm{C}$. The imino chloride was prepared by treating 6 with $\mathrm{POCl}_{3}$ and N,N'-dimethyl aniline at $105{ }^{\circ} \mathrm{C}$. The final coupling was carried out in presence of triethylamine in toluene/THF to afford Int-1 according to Scheme-III.

The reported procedure incorporated during the synthesis of 7-(4-bromobutoxy)-3,4-dihydroquinolin-2(1H)-one (Int$2)^{19}$. Simple acylation carried out on 3 -amino phenol using $\mathrm{NaHCO}_{3}$ in acetonitrile at $0{ }^{\circ} \mathrm{C}$ to afford 10 . The cyclisation of 10 done with $\mathrm{AlCl}_{3}$ in dichloromethane to afford 11. Finally, 11 were coupled with 1,4-dibromo butane using $\mathrm{K}_{2} \mathrm{CO}_{3}$ in DMF at $60^{\circ} \mathrm{C}$ for $12 \mathrm{~h}$ to afford Int-2 according to Scheme-IV.

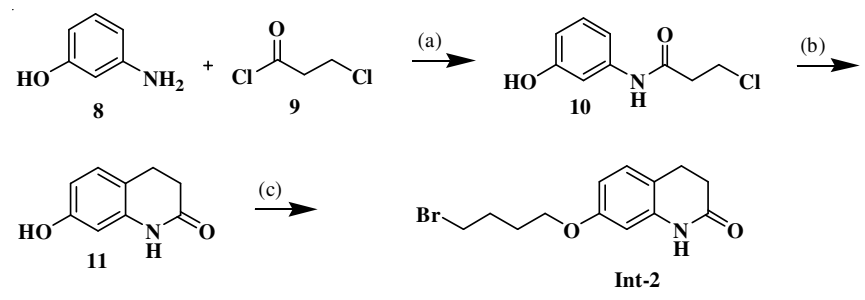

Reagents \& Conditions: (a) $\mathrm{NaHCO}_{3}$ (b) $\mathrm{AlCl}_{3}$ (c) 1,4-dibromo butane

Scheme-IV: Synthesis of 7-(4-bromobutoxy)-3,4-dihydroquinolin-2(1H)one (Int-2)

The traditional approach for the synthesis of Int-3 - Int-7 as shown in Scheme- $\mathbf{V}^{20}$. The conversion of nitro compounds $\mathbf{9}$ and $\mathbf{1 4}$ to corresponding chloro $\mathbf{1 0}$ and $\mathbf{1 5}$ using $\mathrm{POCl}_{3}$ as a chlorinating reagent. The further conversation of choro compounds 10 and $\mathbf{1 5}$ into 11, 16, 17, 22 and $\mathbf{2 7}$ using appropriate alcohols, the methylation of compound 25 using DMS to afford methylated compound $\mathbf{2 6}$. The further conversion of compounds, 11, 16, 17, 22 and 27 to acetate using acetic anhydride to afford compounds, 12, 17, 20, 23 and 28. All these compounds hydrolyzed using $\mathrm{NaOH}$ to afford $\mathbf{1 3}, \mathbf{1 8}$, 21, 24 and 29. Finally, alcohol of 13, 18, 21, 24 and 29 converted to chloro using $\mathrm{SOCl}_{2}$ to afford Int-3 to Int-7 according to Scheme- $\mathbf{V}^{21,22}$.

The earlier reported paper used for the synthesis of Int$8^{23}$. Herein, the required 2-mercapto benzimidazole (Int-8) was prepared by refluxing $o$-phenylenediamine $(\mathbf{3 3}), \mathrm{CS}_{2}$ and 
<smiles></smiles>

(b)<smiles></smiles>

(b)<smiles></smiles><smiles>CCC(=O)OCc1ncc(C)c(OC)c1C</smiles>

12<smiles>COc1c(C)cnc(Cl)c1CO</smiles>

13<smiles>COCCCOc1ccnc(COC(C)=O)c1C</smiles><smiles>COCCCOc1ccnc(Cl)c1CO</smiles>

18<smiles></smiles>

Int-3<smiles>Cc1c([N+](=O)[O-])cc[n+]([O-])c1C</smiles>

14<smiles></smiles>

(b)<smiles>Cc1c(OCC(F)F)cc[n+]([O-])c1C</smiles>
19<smiles></smiles><smiles>CCOc1ccnc(COC(C)=O)c1C</smiles>
20<smiles>CCC(=O)OCc1nccc(OC)c1C</smiles>
23 (d)<smiles></smiles>

21<smiles></smiles>

24<smiles></smiles><smiles></smiles>

Int-5<smiles></smiles><smiles>CCCCCCC(=O)OCc1nccc(OC)c1OC</smiles>
28<smiles></smiles>

29<smiles></smiles>

Int-7

Reagents \& Conditions: (a) $\mathrm{POCl}_{3}$, reflux for 3-4h (b) appropriate alchohols and $\mathrm{NaOH}$ (c)acetic anhydride, acetic acid, $\mathrm{MeOH}$, Toluene (d) $\mathrm{NaOH}, \mathrm{MeOH}, \mathrm{MDC}$ (e) $\mathrm{SOCl}_{2}$ (f) $\mathrm{DMS}$

Scheme-V: Synthesis of 2-chloromethyl-4-methoxy-3,5,-dimethyl-pyridine. HCl (Int-3), 2-chloromethyl-3-methyl-4-(3methyoxypropoxy) pyridine. $\mathrm{HCl}$ (Int-4), 2-chloromethyl-3-methyl-4-(2,2,2-trifluoromethoxy)pyridine. $\mathrm{HCl}$ (Int-5), 2Chloromethyl-3-methyl-4-methoxy pyridine. $\mathrm{HCl}$ (Int-6) and 2-chloromethyl-3,4-dimethoxypyridine. $\mathrm{HCl}$ (Int-7)

$\mathrm{NaOH}$ and further salt freed by using acetic acid according Scheme-VI.

In this process, fluorination of 4-hydroxyacetanilide (34) yielded the intermediate $\mathrm{N}$-[4-(difluoromethoxy)phenyl]acetamide 35. Subsequent reactions like nitration followed by hydrolysis, reduction and cyclization of intermediate $\mathbf{3 5}$ gave the title compound, 2-mercapto-5-difluoromethoxy- $1 H$ benzimidazole Int-9 according Scheme-VII ${ }^{24}$.

The important intermediate for omeprazole, 2-mercapto5 -methoxy benzimidazole (Int 10) ${ }^{23}$ was prepared from anisidine (39), acetyl protection leads $\mathbf{4 0}$. The Int 40 , converted into

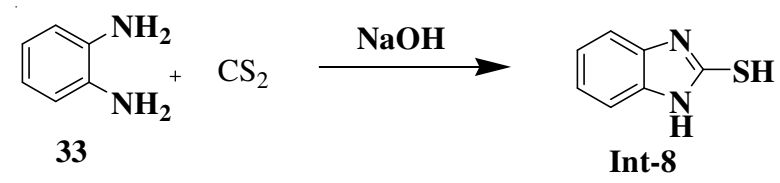

Scheme-VI: Synthesis of 2-mercapto benzimidazole nitro compound 41 using $\mathrm{HNO}_{3}, \mathrm{H}_{2} \mathrm{SO}_{4}$. Then deprotected $\mathbf{4 1}$ into 42 using $\mathrm{NaOH}, \mathrm{MeOH}$. The nitro group reduced using tin, $\mathrm{HCl}$ and further cyclized using $\mathrm{KOH}, \mathrm{CS}_{2}$ to afford Int 10 according to Scheme-VIII.

The coupling of $\mathbf{4 4}$ with sodium methoxide in methanol and further reduction and cyclisation leads Int-11 according to Scheme-IX $\mathbf{X}^{25}$.

The reaction of 2-mercapto-5-nitrobenzimidazole 47 with iron and concentrated $\mathrm{HCl}$ in refluxing ethanol and water gives monoamine 48, which on condensation with 2,5-dimethoxytetrahydrofuran 49 in acetic acid yields 2-mercapto-5-(1pyrrolyl)benzimidazole Int-12 according to Scheme- $\mathbf{X}^{26}$.

The novel targets (SLN11-SLN20) were synthesized by simple coupling using different technologies (microwave, ultra-sonication and normal conventional method). Basically, we observed ultra-sonication condition looking better comparatively with other techniques used based on yield according to Table-1. 
<smiles>CC(=O)Nc1ccc(O)cc1</smiles>

34

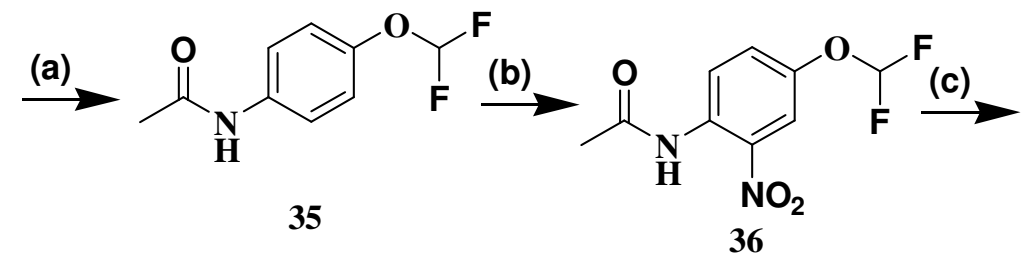<smiles>Nc1ccc(OC(F)F)cc1[N+](=O)[O-]</smiles>

37<smiles>Nc1ccc(OC(F)F)cc1N</smiles>

38

Reagents \& conditions:

(a) PEG-600, difluoromethylenchloride, 50-55 ${ }^{0} \mathrm{C}, 70-74 \mathrm{~h}$ (b) $\mathrm{HNO}_{3}, \mathrm{H}_{2} \mathrm{SO}_{4}, 20-25^{\circ} \mathrm{C}, 2 \mathrm{~h}$.(c) CH3OH, Reflux, 3h. (d) Raney-Nickel, hydrazine hydrate, reflux, 4h. (e) $\mathrm{CS}_{2}$, reflux, $4 \mathrm{~h}$.

Scheme-VII: Synthesis of 6-(difluromethoxy)-1H-benzo[d]imidazole-2-thiol (Int-9)<smiles>COc1ccc(N)cc1</smiles>

39

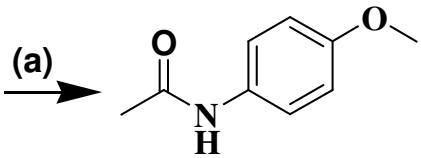

40

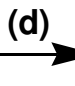

42<smiles>COc1ccc(N)c([N+](=O)[O-])c1</smiles><smiles>COc1ccc(N)c(N)c1</smiles>

43

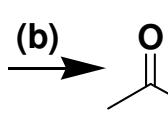<smiles>COc1ccc(N)c([N+](=O)[O-])c1</smiles>

41<smiles>COc1ccc2nc(S)[nH]c2c1</smiles>

Int-10

Reagents \& Conditions: (a) acetic anhydride, acetic acid (b) $\mathrm{HNO}_{3}, \mathrm{H}_{2} \mathrm{SO}_{4}$ (c) $\mathrm{NaOH}, \mathrm{MeOH}$ (d) $\mathrm{Sn}, \mathrm{HCl}$ (e) $\mathrm{CS}_{2}, \mathrm{KOH}, \mathrm{EtOH}$

Scheme-VIII: Synthesis of 6-methoxy-1H-benzo[d]imidazole-2-thiol (Int-10)

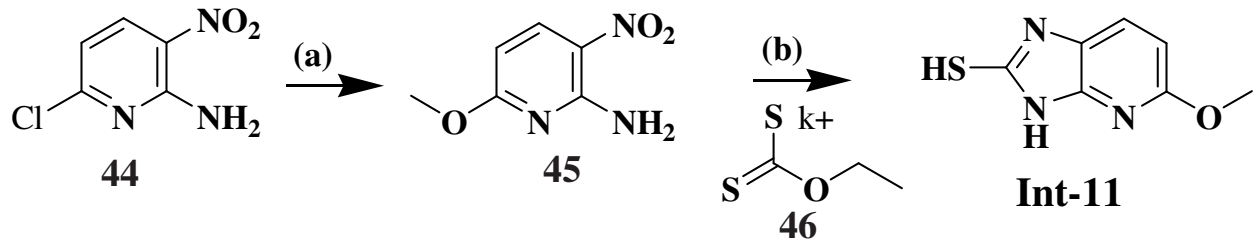

Reagents \& Conditions: (a) $\mathrm{NaOMe}, \mathrm{MeOH}$ (b) Ra-Ni, $\mathrm{H}_{2}$, EtOH

Scheme-IX: Synthesis of 5-methoxy-3H-imidazo[4,5-b]pyridine-2-thiol (Int-11)of 5-methoxy-3H-imidazo[4,5-b]pyridine-2-thiol (Int-11)<smiles>O=[N+]([O-])c1ccc2[nH]c(S)nc2c1</smiles>

47 (a)<smiles>Nc1ccc2[nH]c(S)nc2c1</smiles>

48

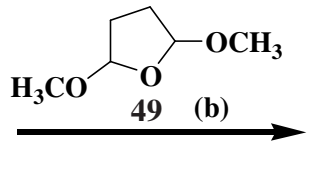<smiles>Sc1nc2cc(-n3cccc3)ccc2[nH]1</smiles>

Reagents \& Conditions: (a) $\mathrm{Fe}, \mathrm{Con} \cdot \mathrm{HCl}$, EtOH, $\mathrm{H}_{2} \mathrm{O}$ (b) $\mathrm{AcOH}$

Scheme-X: Synthesis of 5-(1H-pyrrol-1-yl)-1H-benzo[d]imidazole-2-thiol (Int-12) 
TABLE-1

RESULTS OF TARGETS (SLN11-SLN20)

\begin{tabular}{|c|c|c|c|c|c|}
\hline Entry-1 & Entry-2 & Product & $\begin{array}{c}\text { Conventional method } \\
\text { yield }(\%)\end{array}$ & $\begin{array}{c}\text { Microwave method } \\
\text { yield }(\%)\end{array}$ & $\begin{array}{c}\text { Sonication method } \\
\text { yield (\%) }\end{array}$ \\
\hline Int-1 & Int-3 & SI & 52 & 50 & 70 \\
\hline Int-1 & Int-4 & SLN- & 50 & 49 & 65 \\
\hline Int-1 & Int-5 & SLN- & 49 & 49 & 55 \\
\hline Int-1 & Int-6 & SLN & 42 & 39 & 49 \\
\hline Int-1 & Int-7 & $\mathrm{LN}$ & 50 & 49 & 55 \\
\hline Int-2 & Int-8 & SLN-16 & 49 & 45 & 55 \\
\hline Int-2 & Int-9 & LN-17 & 39 & 41 & 47 \\
\hline Int-2 & Int-10 & SLN-18 & 46 & 43 & 52 \\
\hline Int-2 & Int-11 & SLN-19 & 35 & 35 & 45 \\
\hline Int-2 & Int-12 & -20 & 41 & 39 & 48 \\
\hline
\end{tabular}

\section{Conclusion}

The quinoline and dibenzo thiazepine derivatives were (SLN11-SLN20) successfully synthesized by using literature procedure. The derivatives developed based on drug candidates, prepared skeleton. Simple convergent methodology worked for getting good yields overall. The final, $\mathrm{C}-\mathrm{N}$ and $\mathrm{C}-\mathrm{S}$ coupling approached three techniques, In this regard, sonication tech- nique is good for getting good yield and time. In almost all reactions happened more spots in microwave reaction may be due to microwave other bonds also dislocated and afford low yield. The same conventional reaction yield shown less and taking long time. The explosive reactions, like azide and Mitsunobu reactions, etc. are not useful for bulk scale. 


\section{ACKNOWLEDGEMENTS}

The authors acknowledged the Osmania University for providing the research facility and the direct contributions for the staff of Department of Chemistry and Analytical team.

\section{REFERENCES}

1. M. Morri and N. Takeguchi, J. Biol. Chem., 268, 21553 (1993).

2. H. Fujisaki, H. Shibata, K. Oketani, M. Murakami, M. Fujimoto, T. Wakabayashi, I. Yamatsu, M. Yamaguchi, H. Sakai and N. Takeguchi, Biochem. Pharmacol., 42, 321 (1991).

3. B. Wallmark and H. Larsson, J. Biol. Chem., 260, 13681 (1985).

4. http://www.rxlist.com/prevacid-drug.htm\#cp.

5. http://en.wikipedia.org/wiki/lansorpazole.

6. K. Bernhard, S. Ernst, S.B. Joerg, W.S. Alexander, U. Krueger, S. Hartmann, R. Georg, F. Volker and K. Kurt, J. Med. Chem., 35, 1049 (1992).

7. B. Kohl, E. Sturm and G. Rainer, US 4758579 (1988).

8. H. Nakai, Y. Shimamura, T. Kanazawa, S. Yasuda and M. Kayano, J. Chromatogr. B: Biomed. Appl., 211, 660 (1994).

9. H. Fujisaki, H. Shibata, K. Oketani, M. Murakami, M. Fujimoto, T. Wakabayashi, I. Yamatsu, M. Yamaguchi, H. Sakai and N. Takeguchi, Biochem. Pharmacol., 42, 321 (1991).

10. N. Takeguchi, T. Yamanouchi, H. Sakai and M. Mora, J. Physiol. (Japan), 42, 75 (1992).
11. Belgium Patent No. 549,420.

12. J. Galmiche, S. Bruley Des Varannes, P. Ducrotté, S. Sacher-Huvelin, F. Vavasseur, A. Taccoen, P. Fiorentini and M. Homerin, Aliment Pharmacol. Ther., 19, 655 (2004).

13. T. Muro and T. Muro, Phenyl-Methylthio)ethyl-Piperazines and Related Compounds, US patent 3,238,209, March (1966)

14. (a) R. Sanghi, Resonance, 5, 77 (2000); (b) V. Sridar, Curr. Sci., 74, 446 (1998).

15. D. Wei, X. Yu and X.G. Qing, Chin. Chem. Lett., 16, 327 (2005).

16. S.D. Lepore and Y. He, J. Org. Chem., 68, 8261 (2003).

17. F. Pisani, A. Fazio, C. Artesi, M. Russo, R. Trio, G. Oteri, E. Perucca and Di Perri, J. Neurol. Neutosurg. Psychiatry, 55, 126 (1992).

18. D.M. Ganesh, M.K. Yogesh, Ashok Kumar, Dharmendra Singh, M.K. Kishan and B.W. Suresh, Indian J. Chem., 50B, 1196 (2011).

19. J.A. VanAllan and B.D. Deacon, Org. Synth., 4, 569 (1963).

20. K.B. Sharpless and V.R. Thomas, Aldrichim. Acta, 12, 63 (1979).

21. US Patent, 4, 628,098, Dec. (1986).

22. J.A. VanAllan and B.D. Deacon, Org. Synth., 4, 569 (1963).

23. S.S. Mahajan and R.G. Nandre, Indian J. Chem., 45B, 1756 (2006).

24. J.J. Vora, K.P. Trivedi and R.S. Kshatriya, Adv. Appl. Sci. Res., 2, 89 (2011).

25. G. Thomas and S. Sepehr, WO/2008/127640.

26. S. Sripathi, B.R. Reddy, K.V. Reddy, V.V.N.K.V. Raju and D.K. Mayur, Org. Process Res. Dev., 13, 804 (2009). 